\title{
Research on Vehicle Routing Algorithm for Supply Chain Logistics Distribution
}

\author{
Hao Zou ${ }^{1,2}$ and Tiantian Zhang ${ }^{1}$ \\ ${ }^{1}$ Sichuan Special Equipment Inspection and Research Institute, Chengdu, Sichuan, 610061 China \\ ${ }^{2}$ School of Public Administration, University of Electronic Science and Technology of China, Chengdu, Sichuan, 610054 China
}

Keywords: Planning algorithm; Supply chain management; Vehicle routing; Path planning;

\begin{abstract}
In view of logistics distribution vehicle scheduling problem, this paper proposes a vehicle routing optimization model considering route and time. Secondly, the vehicle routing optimization model is designed in detail, and the mathematical derivation steps are given, and the decision-making process of this problem is simulated with data. Finally, the paper takes the route of logistics distribution vehicles as the goal, and makes a preliminary verification. The results show that the algorithm is conducive to improving the management efficiency of the whole supply chain.
\end{abstract}

\section{Introduction}

Logistics distribution is an important link in the supply chain. It is the process of delivering goods from logistics nodes to consignees. Research shows that transportation costs account for about half of the total logistics. Therefore, it is very important to reasonably and effectively distribute vehicle scheduling and vehicle routing to reduce transportation costs. Vehicle optimal scheduling includes line optimization, cargo loading and delivery line optimization. In foreign countries, similar work has been widely used in all aspects of production and life, such as the optimization of newspaper delivery and line, the optimization of milk distribution and delivery lines, the vehicle loading and line design, the optimization of the line of the garbage truck, the delivery of the chain stores and the optimization of the lines. This paper will study the path planning algorithm in logistics distribution.

\section{The working flow of the whole system}

The workflow of the system is: the user input the demand information of the delivery point, then the system choose different scheduling mode after passing the validity test, and transfer the data to the transport plan generation system. The arithmetic module checks and calculates the transmitted data, and displays the results and statistical indicators in the form of text. Finally, the user can display the route on the electronic map to see if he is satisfied with the scheduling plan. The workflow is shown in Figure 1.

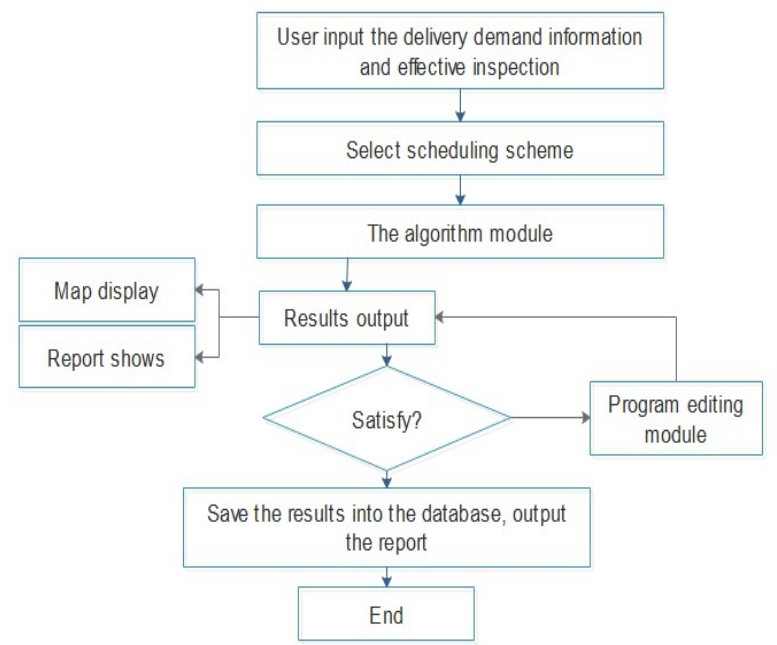

Figure 1. The working flow chart of the whole system

\section{Route planning algorithm for logistics distribution vehicle in supply chain}

Logistics distribution is an important link directly connected with consumers in the supply chain, and is the process of goods arriving at the consignee from the logistics node. The research shows that the transportation cost accounts for about $1 / 2$ in the whole logistics, so it is very important to plan a reasonable and effective distribution vehicle scheduling and vehicle route to 
reduce the transportation cost.Distribution vehicle scheduling optimization, including the optimization of freight collection route, freight loading and delivery route optimization, as well as the integration of freight collection, distribution and delivery optimization.

In order to construct the mathematical model, the distribution center number is 0 , and the customer demand number is $1 . \mathrm{n}$, customer point and distribution center are all $i(i=0, \ldots n)$, to show it. The parameter variables are defined as follows:

$\mathrm{n}$ :Total demand customer number;

Cij:Transport cost of point $\mathrm{i}$ to point $\mathrm{j}$;

$\mathrm{q}:$ Vehicle number limit

di:quantity demanded;

$\mathrm{yjk}=1$ :The requirement point $\mathrm{i}$ is delivered by the

vehicle $\mathrm{k}$, otherwise it is 0 ;

$\mathrm{xijk}=1$ : distributed by vehicle $\mathrm{k}$ from demand point $\mathrm{i}$ to the demand point $\mathrm{j}$, otherwise it will be 0 .

ETi: Earliest start service time

LTi: Service time at the latest

ti: Earliest start service time of point i;

Si: service time of point $i$;

tij: driving time from point $\mathrm{i}$ to point $\mathrm{j}$;

bi: The arrival time of the vehicle

Ce: Opportunity cost of waiting at the early arrival of a vehicle

C1:A penalty per unit time when a vehicle is late

The specific operation steps are as follows:

1. logistics distribution database selects the road map of the location where the distribution center is located.

2. Mark the specific geographical location of each customer on the map, and form a distribution point (including distribution center) layer, overlay with the road network layer.

3. using ArcMap's network analysis toolbar, Utility Network analyst, the shortest path analysis function:

(1)We look for the shortest path of the distribution center and customer location in the graphic window, and click to place the flag.

(2)Set the element weight of the network connection path in the weight field.

(3)Find shortest Path and Solve Button,calculate the minimum transportation cost between any distribution center and customer point..

(4)In geographic information system, the average speed is generally determined according to the grade of roads.

(5)Setting up a time window model.

So, the main objective function changes are as follows:

$$
\operatorname{Minz}=\sum_{i} \sum_{j} \sum_{k} C_{i j} x_{i j k}+C_{e} \sum_{j=1}^{n} \max \left(E T_{j}-b_{j}, 0\right)+C_{1} \sum_{j=1}^{m} \max \left(b_{j}-L T_{j}, 0\right)
$$

The transport cost $\mathrm{Cij}$ is equal to the product of the shortest path distance and the unit kilometre. Ce and $\mathrm{C} 1$ are respectively fines for opportunity cost and delay cost. $\mathrm{Ce}, \mathrm{C} 1$ and weighted speeds can be compared:

A) if the $\mathrm{Ce}$ and $\mathrm{C} 1$ are much smaller than the $5 \times$ weighted average speed, then the objective function can be simplified:

$$
\operatorname{Minz}=\sum_{i} \sum_{j} \sum_{k} C_{i j} x_{i j k}
$$

B) if the $\mathrm{Ce}$ and $\mathrm{C} 1$ are much larger than the 5* weighted average speed, then the objective function can be simplified:

$$
\operatorname{Minz}=C_{e} \sum_{j=1}^{n} \max \left(E T_{j}-b_{j}, 0\right)+C_{1} \sum_{j=1}^{m} \max \left(b_{j}-L T_{j}, 0\right)
$$

(6) Run C-W saving heuristics program solution.

(7) Return the results of vehicle routing to the Geographic Information System (GIS), and display the routes in the form of map report with Crystal report module in Am/lnfo.

\section{Model simulation analysis}

There are many trucks in the distribution center, the maximum load is 10 tons. Drivers work from 8 a.m. to 6 p.m. One day, the manager received an order from 13 customers (number 1,.. 13), the location of each customer is specifically marked on the map, the demand of each customer di $(0<\mathrm{di}<10)$, the service time of each customer $\mathrm{Si}$ (the customer service time is proportional to the customer's demand), and the time range of each customer's permission to execute the service [ETi, LTi] $(8<\operatorname{ETi}(\mathrm{LTi}<18)$ is given by the lower table 1 .

Table 1. Customer demand and service time requirements

\begin{tabular}{c|c|c|c|c|c|c|c|c|c|c}
\hline Customer & 1 & 2 & 3 & 4 & 5 & 6 & 7 & 8 & 9 & 10 \\
\hline $\mathrm{d}_{\mathrm{i}}$ (Tons of) & 2.4 & 1.8 & 2.2 & 4.6 & 3 & 0.8 & 2.8 & 1.6 & 3 & 2.6 \\
\hline $\mathrm{s}_{\mathrm{i}}$ & 1.2 & 0.9 & 1.1 & 2.3 & 1.5 & 0.4 & 1.4 & 0.8 & 1.5 & 1.3 \\
\hline $\mathrm{Et} \perp \mathrm{I}_{\mathrm{i}}$ & $8,10.5$ & 9,11 & 9,12 & 13,17 & 8,11 & 13,15 & 14,17 & $9,11.5$ & 13,16 & 9,13 \\
\hline
\end{tabular}

Run $\mathrm{C}-\mathrm{W}$ saving heuristics program solution. The result of the delivery arrangement is as follows: 2 .

Table 2. Vehicle routing arrangement

\begin{tabular}{c|c|c|c|c}
\hline Truck & Route & Length $(\mathrm{km})$ & Loading capacity (ton) & Loading rate \\
\hline 1 & $0-1-3-4-0$ & 78 & 9.2 & $92 \%$ \\
\hline 2 & $0-2-9-0$ & 52 & 3.3 & $33 \%$ \\
\hline
\end{tabular}




\begin{tabular}{l|c|c|c|c}
3 & $0-8-12-6-7-0$ & 98 & 9.8 & $98 \%$ \\
\hline 4 & $0-5-10-12-13-0$ & 112 & 10 & $100 \%$ \\
\hline
\end{tabular}

We make use of 4 cars distribution vehicle, the total distance of vehicles is 340 kilometers, the total route transportation cost equals 1700 yuan. This is consistent

with the result of the best route arrangement using the exact optimization method. The time to reach the customer points is shown in Table 3 below:

Table 3. Line timetable

\begin{tabular}{c|c|c|c|c}
\hline Truck & 1 & 2 & 3 & 4 \\
\hline Route & $0-1-3-4-0$ & $0-2-9-0$ & $0-8-11-6-7-0$ & $0-5-10-12-13-0$ \\
\hline Time & $8-805-10-11.3-15.9$ & $8-8.3-11.3-15.9$ & $8-8.3-10.1-16.9$ & $8-8.6-10.5-12.1-14.6-16.2$ \\
\hline
\end{tabular}

The cost of waiting time is 315 , and the total delivery cost is 2015.Therefore, the algorithm can calculate the minimum waiting time and minimum cost to meet the design requirements.

\section{Conclusions}

The supply chain is a wider operation structure than the enterprise. It involves many links from suppliers to customers. How to improve the competitiveness of the whole supply chain to face the changeable market environment, to face the fierce competition of globalization, has become a hot research topic in today's society. The paper have studied and explored the direction of optimizing the logistics distribution and improving the benefit of the supply chain. It is helpful for the enterprises to use the existing resources effectively and reduce the cost.In fact, with the development of E-commerce, logistics and GIS, GIS technology will become an indispensable part of supply chain management.

\section{References}

1. Liu Xiaoyan. Application of Activity-Based Costing in cost accounting of third party logistics enterprises [J]. Time Education, 2014 (5): 92-94

2. Xu Hong, Liu Zhiqiang, LuoJie. Design of logistics distribution system based on GIS[J]. Application Research of Computers, 2002 (6): 103-106

3. Zhang Qi, Pan Hongping. Analysis of supply chain structure model of integrated GIS[J]. Logistics technology, 2005 (3):29-31

4. Xia Jiong. Cost accounting of the three party logistics enterprises based on Activity-Based Costing [J]. Communication of Finance and Accounting, 2010 (110:27 6-278)

5. Hu Lingling. Application of Activity-Based Costing in cost management of third party logistics enterprises [J]. Logistics platform, 2010(7):121-124 